I BIENAL LATINOAMERICANA DIDH

\title{
Interdependencia e indivisibilidad de los derechos humanos: Una nueva mirada frente al covid-19
}

\author{
Interdependence and indivisibility of human rights: A renewed glance \\ in the face of Covid-19
}

\author{
Flávia Piovesan \\ Pontificia Universidad Católica de São Paulo, Brasil \\ Mariela Morales Antoniazzi \\ Instituto Max Planck de Derecho Público Comparado \\ $y$ Derecho Internacional de Heidelberg, Alemania
}

\begin{abstract}
RESUMEN La pandemia de covid-19 y sus efectos multidimensionales demandan reforzar un enfoque holístico de los derechos humanos, que gire en torno a su interdependencia e indivisibilidad. El argumento se centra en ofrecer una dogmática jurídica reinterpretativa de reforzamiento «a la inversa» de ambos principios, en la medida que la salvaguarda de los derechos económicos, sociales, culturales y ambientales (DESCA) requiere también la garantía de los derechos civiles y políticos. El carácter reforzado deriva de la urgencia de atender a las personas y grupos en situación de vulnerabilidad. $\mathrm{El}$ artículo dialoga con la base teórica del ius constitutionale commune en derechos humanos emergente para enfrentar la pandemia. Sobre la base del método bibliográfico, el argumento se desarrolla siguiendo tres momentos, a saber: en el punto de partida pre covid-19 de la propia Declaración Universal y los instrumentos regionales de protección de los derechos humanos en el caso de América Latina; la interpretación a la inversa frente al covid-19 en razón de los estándares universales, regionales y nacionales, tomando el caso argentino como referencia; y el discurso académico pos covid-19, para afianzar el lenguaje de la indivisibilidad e interdependencia como ruta para apalancar las profundas transformaciones. Los retos actuales revelan el potencial de los derechos humanos como lenguaje para guiar a los Estados hacia el cumplimiento de los estándares que conforman la base del nuevo ius commune pospandemia.
\end{abstract}

PALABRAS CLAVE Interdependencia, indivisibilidad, interpretación inversa, ius constitutionale commune. 


\begin{abstract}
The Covid-19 pandemic and its multidimensional effects demand the reinforcement of a holistic approach to human rights, centered on their interdependence and indivisibility. The article advocates for a legal dogmatic reinterpretation and a "reverse" strengthening of both principles arguing that safeguarding economic, social, cultural and environmental rights (ESCER) also requires the guarantee of civil and political rights. The reinforced character stems from the urgency of attending people and groups in a situation of vulnerability. The article discusses the theoretical base of the emerging ius constitutionale commune on human rights to confront the pandemic. Based on the bibliographic method, the argument is developed in three stages: in the pre-covid-19 stage of the Universal Declaration itself and the regional instruments for the protection of human rights in the case of Latin America; the reverse interpretation in face of covid-19 in terms of the universal, regional and national standards, taking the Argentine case as a reference; and the post-covid-19 academic discourse, to reinforce the language of indivisibility and interdependence as a route to gain leverage for profound transformations. The current challenges reveal the potential of human rights as a language to guide States towards compliance with the standards that form the basis of the new postpandemic ius commune.
\end{abstract}

KEYWORDS Interdependence, indivisibility, reverse interpretation, ius constitutionale commune.

\title{
Introducción
}

El covid-19 y sus efectos negativos en la garantía de los derechos humanos se revelan a nivel global en cualquier faceta de la condición humana. Es incuestionable, por tanto, la reafirmación de los derechos humanos, que «son universales, indivisibles e interdependientes, están relacionados entre sí y se refuerzan mutuamente para la creación o reconstrucción de sociedades resilientes, inclusivas, justas y pacíficas», como declaró en julio de 2020 la Asamblea General de las Naciones Unidas con ocasión del $75 .^{\circ}$ aniversario de la Organización. ${ }^{1}$

El presente análisis propone repensar y reevaluar los principios de indivisibilidad e interdependencia de los derechos, a la luz del nuevo desafío emergente para la vigencia de todos los derechos humanos frente a la pandemia. Ello implica, por una parte, un ejercicio de dogmática jurídica reinterpretativa y, por la otra, un enfoque innovador e interdisciplinario que contribuya a comprender el alcance de una crisis multidimensional, multinivel y con multiplicidad de consecuencias devastadoras en todas las esferas de la vida, y en particular, con repercusiones desproporcionadas en

1. «Follow-up to and implementation of the Vienna Declaration and Programme of Action», Consejo de Derechos Humanos, Naciones Unidas, A/HRC/44/L.17/REV.1, 14 de julio de 2020, disponible en https://bit.ly/32Z23vQ. 
las personas y grupos en situación de vulnerabilidad. Como lo afirma el secretario general de Naciones Unidas, el virus no discrimina, pero sus impactos sí. ${ }^{2}$

Se trata de un momento histórico en el que la normativa vigente en tiempos de normalidad está sometida a escrutinio. ${ }^{3}$ La situación actual representa una llamada de atención y un recordatorio sobre la necesidad de garantizar los derechos y el acceso a los servicios básicos para toda la población cuando no se está en tiempos de emergencia, pues solo bajo esa condición se puede garantizar su protección durante una emergencia. ${ }^{4}$ El covid-19 es una prueba para las sociedades, los Gobiernos, las comunidades y los propios individuos, en tanto el respeto de los derechos humanos en todo su espectro será fundamental para el éxito de la respuesta de salud pública y la recuperación de la pandemia, como lo destaca la Oficina de la Alta Comisionada para los Derechos Humanos de Naciones Unidas.

En la comunidad de práctica de los derechos humanos (von Bogdandy y Urueña, 2020) hay convergencia en identificar que la crisis de la pandemia pone de relieve una vez más el carácter indivisible e interdependiente de los derechos humanos. ${ }^{5}$ Asimismo, se constata un acuerdo en instar a los Estados a que las medidas adoptadas que puedan afectar o restringir el goce y ejercicio de derechos humanos deben cumplir los principios de temporalidad, legalidad, razonabilidad, necesariedad y proporcionalidad, al igual que los objetivos definidos según criterios científicos, en aras de cumplir los principios del Estado de derecho y la democracia.

El argumento que se sostiene en este análisis consiste en afirmar que la pandemia ha generado una interpretación reforzada «a la inversa» de la interdependencia e indivisibilidad de los derechos humanos, en la medida que la salvaguarda de los derechos económicos, sociales, culturales y ambientales (DESCA) requiere la garantía de los derechos civiles y políticos, no solo a la inversa. ${ }^{6}$ En la lectura tradicional se invocaba con frecuencia la interdependencia e indivisibilidad para justificar la justiciabilidad directa de los DESCA en función de la necesidad de la misma garantía que los derechos civiles y políticos.

2. António Guterres, «We are all in this together: Human rights and Covid-19 response and recovery», Naciones Unidas, 23 de abril de 2020, disponible en https://bit.ly/2Gajr8g.

3. Brett Milano, «Restricting civil liberties amid the Covid-19 pandemic», Harvard Law Today, 21 de marzo de 2020, disponible en https://bit.ly/2GaTaGU.

4. Nate Schenkan, «Covid-19 and the erosion of human rights», Freedom House, 27 de julio de 2020, disponible en https://bit.ly/2G8Na12.

5. Véase Human Rights Center, «Human rights dimensions of Covid-19», University of Dayton Blogs, 23 de marzo de 2020, disponible en https://bit.ly/2S9OaVL; Melik Özden, «Covid-19 pandemic and human rights», CETIM, 29 de abril de 2020, disponible en https://bit.ly/34crycP.

6. Flávia Piovesan y Mariela Morales Antoniazzi, «Covid-19 and the need for a holistic and integral approach to human rights protection: On Latin America and the inverted principle of interdependence and indivisibility of human rights», Verfassunbsblog, 25 de abril de 2020, disponible en https://bit.ly/2Dif4qn. 
Frente a la pandemia, esta nueva mirada interpretativa de la interdependencia e indivisibilidad rige para la protección de los DESCA (salud, agua, educación, trabajo), y también para garantizar los derechos civiles y políticos (libertad de expresión, acceso a la información, garantías judiciales). El carácter reforzado deviene de la dimensión de la interseccionalidad $-\mathrm{y}$, en consecuencia, de la urgencia dentro de la emergencia - de atender a las personas y grupos en situación de vulnerabilidad.

El artículo dialoga con la base teórica del ius constitutionale commune en derechos humanos emergente para enfrentar la pandemia en clave de los pronunciamientos de los órganos de derechos humanos de Naciones Unidas y las Américas, al igual que la normativa nacional, que muestran la tendencia a definir la lucha contra el covid-19 como una batalla por los derechos humanos, y configuran un ius commune con los estándares establecidos.

El argumento de la interpretación reforzada «a la inversa» se presenta en tres pasos, partiendo del método bibliográfico. Primero, se exponen ambos principios en el punto de partida pre covid-19 de la Declaración Universal y los instrumentos regionales de América Latina. Segundo, se aborda la nueva lectura de estos principios enfatizando la interpretación a la inversa frente al covid-19, respecto de los estándares universales, regionales y nacionales, tomando el caso argentino como referencia. Tercero, se esboza el potencial del discurso académico para afianzar el lenguaje de la indivisibilidad e interdependencia como ruta para las profundas y necesarias transformaciones pos covid-19. Se concluye con unos comentarios acerca de los ejes transversales del acervo común de la humanidad para afrontar la nueva realidad.

\section{Reconstrucción de la interdependencia e invisibilidad: Las Declaraciones como punto de partida pre covid-19}

La Declaración Universal de Derechos Humanos de 1948 contiene una gramática innovadora, ya que introdujo la idea contemporánea de indivisibilidad y universalidad de los derechos y dio lugar a la redacción del Pacto Internacional de Derechos Civiles y Políticos (ICCPR) y del Pacto Internacional de Derechos Económicos, Sociales y Culturales (PIDESC). La universalidad se reconoce como la piedra angular del derecho internacional de los derechos humanos y dispone que las libertades fundamentales y los derechos humanos deben aplicarse a todos y en cualquier lugar, bajo la convicción de que la condición de persona es el único requisito para la dignidad y titularidad de derechos. «Solo el reconocimiento integral de todos [los] derechos puede asegurar la existencia real de cada uno de ellos» (Gros Espiell, 1986: 16-17). La indivisibilidad como lenguaje se utiliza tradicionalmente para describir la igualdad fundamental de los derechos contenidos en ambos pactos, a pesar de las diferencias en cuanto a sus obligaciones. No obstante, en sentido más estricto, la indivisibilidad pretendía reforzar la relevancia de los derechos económicos y sociales frente a la per- 
cepción de que la división de la «unidad fundamental» de la Declaración Universal priorizaba los derechos civiles y políticos a expensas de los derechos económicos y sociales. La interdependencia, en esencia, se refiere a la medida en que «dos o más derechos o grupos de derechos pueden depender entre sí para su efectividad» (Mihr, 2014: 123).

Desde las Naciones Unidas, en particular de la Oficina del Alto Comisionado, se ha insistido en que los derechos humanos son inherentes a la dignidad de cada persona. En consecuencia, todos los derechos humanos tienen el mismo estatus, no pueden ser colocados en un orden jerárquico y mantienen una interrelación e interdependencia. La negación de un derecho infaliblemente impide el disfrute de otros derechos. Si la privación de un derecho afecta de manera negativa los demás derechos, también la efectiva garantía de un derecho facilita el avance en la salvaguarda de los demás.

La interdependencia e indivisibilidad no solo ha sido reiterada por las principales resoluciones de la Asamblea General de las Naciones Unidas, ${ }^{7}$ sino también en 1993, con la solemne Declaración y el Programa de Acción de Viena, que pone otra vez de relieve que los derechos humanos «están relacionados entre sí, son interdependientes y se refuerzan mutuamente, y que deben tratarse de manera justa y equitativa, en pie de igualdad y asignándoles la misma importancia».

Los principios de indivisibilidad e interdependencia de los derechos humanos se han recogido en diversos instrumentos regionales. La Declaración Americana de los Derechos y Deberes del Hombre (DADH), adoptada en la Novena Conferencia Internacional Americana en abril de 1948 - ocho meses antes de la DUDH—, fue precisamente la primera enumeración detallada de derechos adoptada por una organización intergubernamental (Farer, 1997). Tanto la Comisión Interamericana de Derechos Humanos (CIDH) como la Corte Interamericana de Derechos Humanos (Corte IDH), ejerciendo su mandato transformador en la protección y defensa de los derechos humanos, han construido un corpus iuris interamericano que representa un avance histórico en la noción de interdependencia e indivisibilidad. Solo a título enunciativo, se puede mencionar la jurisprudencia sólida y progresiva en la justiciabilidad directa de los DESCA, entre otros, en el caso Lagos del Campo con Perú, que reconoció por primera vez una violación directa del artículo 26 de la Convención Americana de Derechos Humanos (CADH); el caso Poblete Vilches y otros con Chile (Piovesan, Morales Antoniazzi y Montero, 2019), que advirtió sobre

7. Véanse las Resoluciones de la Asamblea General 40/114, del 13 de diciembre de 1985; 41/117, del 4 de diciembre de 1986; 42/102, del 7 de diciembre de 1987; y 43/113, del 8 diciembre de 1988, todas con el título «Indivisibilidad e interdependencia de las derechos culturales, civiles y políticos», además de una serie de resoluciones de la Asamblea General sobre pactos internacionales de derechos humanos. Véase, por ejemplo, la Resolución 60/149, del 16 de diciembre de 2005. 
la necesidad de garantizar el derecho a la salud sin discriminación; y el caso Cuscul Pivaral y otros con Guatemala, en que la Corte señaló que los Estados, al promover el derecho a la salud, deben "prestar especial atención a los grupos vulnerables y marginalizados».

El caso Lhaka Honhat ${ }^{8}$ representa un hito en la jurisprudencia interamericana, más aún en el contexto de la pandemia, porque por primera vez se pronuncia sobre la violación de manera autónoma de los DESCA que atañen a pueblos y comunidades indígenas y declaran vulnerados, entre otros, el derecho al medio ambiente sano, a la alimentación y al agua. Con el enfoque centrado en las víctimas, el Sistema Interamericano, que gira en la órbita de salvaguardar la dignidad humana y prevenir el sufrimiento humano, el intenso debate sobre la justiciabilidad directa de los DESCA dio una particular visibilidad a los principios de interdependencia e invisibilidad en América Latina.

En el ámbito europeo, la noción de indivisibilidad, interdependencia e interrelación se encuentra de manera implícita en el Preámbulo del Convenio Europeo de Derechos Humanos (CEDH) de 1950 (Koch, 2009: 2), que se inscribe como un instrumento que da «los primeros pasos para la aplicación colectiva de algunos de los derechos enunciados en la Declaración Universal». El CEDH se centra sobre todo en los derechos civiles y políticos, pero hay dos rasgos distintivos en Europa que van en la línea de la indivisibilidad e interdependencia. En primer lugar, la Carta Social Europea de 1961 y la Carta Social Europea Revisada de 1996 comprenden ampliamente los derechos económicos, sociales y culturales. En segundo lugar, los límites del Convenio se ven trascendidos en las prácticas institucionales, ya que la indivisibilidad se ha consolidado como "un principio normativo de trabajo» (Williams, 2013), tendencia que se nota en la jurisprudencia más reciente (Carmona Cuenca, 2017).

La Carta Africana de Derechos Humanos y de los Pueblos de 1981 se distingue por abarcar en un único instrumento los derechos civiles y políticos, económicos, sociales y culturales y colectivos en África -incluidos los derechos de los grupos y los pueblos-, y reconoce que protegen los derechos humanos sin hacer ninguna distinción entre su justiciabilidad o aplicación (Ssenyonjo, 2016). Desde el propio Preámbulo, la Carta Africana proclama la indivisibilidad, interdependencia e interrelación de todos los derechos.

8. Sentencia del caso Comunidades Indígenas Miembros de la Asociación Lhaka Honhat (Nuestra Tierra) con Argentina, Corte Interamericana de Derechos Humanos, fondo, reparaciones y costas, serie C núm. 400, 6 de febrero de 2020 . 


\section{Reinterpretación de la indivisibilidad e interdependencia: El ius commune emergente frente al covid-19}

La pandemia de covid-19 revela la necesidad de reforzar el enfoque holístico de los derechos humanos. La interdependencia e indivisibilidad rige para la protección de los derechos a la salud, el agua, la educación y el trabajo (DESCA) y para garantizar los derechos civiles y políticos (libertad de expresión, acceso a la información, entre otros). Tal protección debe ser reforzada para atender a las personas y grupos en situación de vulnerabilidad. Solo para tomar un ejemplo, en el contexto del covid-19 se corroboró que el derecho esencial a la salud depende igualmente del derecho a la información como eje clave del derecho a la libre expresión. ${ }^{9}$ De allí que es fundamental la debida consideración de la universalidad, indivisibilidad, interdependencia e interrelación de derechos; la igualdad y no discriminación; la perspectiva de género, diversidad e interseccionalidad; la inclusión; accountability; el respeto al Estado de derecho y el fortalecimiento de la cooperación entre los Estados. Esta mirada implica que las medidas que puedan afectar o restringir el goce y ejercicio de derechos humanos deben cumplir los principios de temporalidad, legalidad, razonabilidad, necesariedad y proporcionalidad, además de estar acorde con criterios científicos.

La pandemia arroja un ejercicio de dogmática jurídica reinterpretativa orientado hacia el acervo emergente del ius commune respecto de los DESCA y la suspensión o restricción de garantías a la luz de su indivisibilidad e interdependencia. Tres preguntas guían esta mirada: i) ¿por qué hace falta una interpretación reforzada de las personas y grupos en situación de vulnerabilidad?; ii) además, en clave multinivel (global y regional y comparado), ¿puede identificarse una renovada vigencia de ambos principios?; iii) en razón de las tendencias, tomando Argentina como caso de estudio, ¿puede perfilarse a nivel nacional una respuesta que se aproxima hacia esta reinterpretación?

En cuanto a la urgencia dentro de la emergencia, se ha demostrado que el virus tiene un impacto diferenciado en grupos históricamente excluidos, vulnerables o de riesgo, ${ }^{10}$ que demandan una protección reforzada. En este sentido, la pandemia

9. La CIDH emitió, entre otros, el comunicado de prensa $\mathrm{R}_{58 / 20}$, en el que subraya que los Estados deben tener como centro el pleno respeto de los derechos humanos, y por tanto tienen la obligación de garantizar a todas las personas el acceso a la información precisa respecto de la naturaleza de la amenaza y de los medios para protegerse de ésta. También resaltó la necesidad de garantizar acceso a internet, de proteger la labor de periodistas, tomar acciones para abordar la desinformación y, sin duda, respetar los derechos humanos en caso de llevarse a cabo tareas de vigilancia mediante herramientas tecnológicas.

10. Entre ellos, personas mayores, personas que sufren enfermedades preexistentes, personas privadas de libertad, mujeres, pueblos indígenas, personas en situación de movilidad humana, niñas, niños y adolescentes, personas LGBTI, personas afrodescendientes, personas con discapacidad, personas trabajadoras y personas que viven en pobreza y pobreza extrema, en especial trabajadoras informales y 
arroja tres grandes desafíos estructurales a afrontar: la desigualdad socioeconómica, los patrones históricos de discriminación y los dilemas concernientes a la institucionalidad democrática. Bastan algunos indicadores de la multidimensionalidad de la crisis que exacerba estos déficits sistémicos y conducen la mirada hacia tales desafíos. Philip Alston, anterior relator especial de Naciones Unidas sobre la pobreza extrema y derechos humanos, afirmó en abril de 2020 que las respuestas al covid-19 le están fallando a las personas en situación de pobreza. ${ }^{11}$ Según los expertos de las Naciones Unidas, «la lucha global contra la pandemia tiene pocas posibilidades de tener éxito si la higiene personal, la principal medida para prevenir el contagio, no está al alcance de los 2.200 millones de personas que no tienen acceso a servicios de agua potable». ${ }^{12}$ Por su parte, el relator especial de Naciones Unidas sobre los derechos de los pueblos indígenas, José Francisco Cali Tzay, declaró que «los estados de emergencia están exacerbando la marginación de las comunidades indígenas». En relación con la institucionalidad democrática, se observa que los Estados han adoptado medidas para salvaguardar la salud, pero han dictado declaratorias de estados de excepción y la suspensión o restricción de derechos, que en muchos casos obstaculizan el ejercicio pleno de la democracia. ${ }^{13}$ Ciertas voces, sin embargo, resaltan la relevancia del control del Ejecutivo por parte del Legislativo y Judicial en esta materia (Ginsburg y Versteeg, 2020). ${ }^{14}$

En lo que concierne a la clave multinivel (global y regional y comparado) para avalar la reinterpretación de la indivisibilidad e interdependencia a la inversa, resulta inexorable examinar - de manera exclusivamente enunciativa- la reiteración y creación de estándares para enfrentar la pandemia en el enfoque de DESCA y derechos civiles y políticos. La selección de los pronunciamientos es estrictamente aleatoria e inacabada, porque excedería los límites de esta reflexión. Se focalizan en mostrar una tendencia. Tanto las Naciones Unidas como los sistemas regionales de derechos hu-

personas en situación de calle, así como defensoras y defensores de derechos humanos, líderes sociales, profesionales de la salud y periodistas.

11. Cepal, la Organización Internacional del Trabajo (OIT), el Banco Interamericano de Desarrollo (BID) y el Banco Mundial han elaborado los escenarios e incluso creado observatorios para monitorear los impactos del covid-19.

12. «No se podrá parar el covid-19 sin proporcionar agua a las personas en situación de vulnerabilidad: Expertos de la ONU», Naciones Unidas, 23 de marzo de 2020, disponible en https://bit.ly/3e6L9PO.

13. A título de ejemplo véase, Tamar Hostovsky Brandes, «Israel's perfect storm: Fighting Coronavirus in the midst of a constitutional crisis», Verfassungsblog, 7 de abril de 2020, disponible en https://bit. ly/3mSu7Jq; Radosveta Vassileva, «Bulgaria: ¿Covid-19 as an excuse to solidify autocracy?», Verfassungsblog, 10 de abril de 2020, disponible en https://bit.ly/309tYaO; Jesús María Hernández Casal y Mariela Morales Antoniazzi, «States of emergency without Rule of Law: The case of Venezuela», Verfassungsblog, 22 de mayo de 2020, disponible en https://bit.ly/2FTdqgq.

14. Andrés Malamud, «¿Y si la pandemia mejora la democracia?», El País, 14 de junio de 2020, disponible en https://bit.ly/3kTf5Bv. 
manos - esta contribución considera solamente el sistema interamericano en América Latina- cuentan con bases de datos, comprensivas de todo el acervo configurado en torno al covid-19. Se enuncian, por tanto, solo algunos ejemplos.

\section{Estándares internacionales: Naciones Unidas}

Los órganos internacionales de derechos humanos han emitido pronunciamientos en torno al enfoque de derechos humanos para hacer frente a la pandemia, como, por ejemplo, dos publicados sobre covid-19 y derechos humanos de las Naciones Unidas $^{15}$ y de la Organización Mundial de la Salud. ${ }^{16}$ El secretario de Naciones Unidas, la alta comisionada, los comités —en particular el Comité DESC - ${ }^{17}$ los relatores y expertos ${ }^{18}$ subrayan la interdependencia e indivisibilidad, y le han dado visibilidad al impacto discriminatorio del virus en las personas y grupos en situación de vulnerabilidad. Las Naciones Unidas están apelando a los Estados para dar cumplimiento a los estándares de protección de los derechos humanos en su integralidad. Incluso la aseveración de la alta comisionada de Derechos Humanos define explícitamente la urgencia de la garantía del derecho a la salud, ya que «la universalidad de la amenaza de covid-19 crea el argumento más convincente que jamás ha existido a favor del acceso universal y asequible a la atención médica», ${ }^{19}$ porque «nadie está a salvo hasta que todos están a salvo $»^{20}$.

En esta línea y de forma innovadora, el 9 de abril de 2020, el Consejo de Derechos Humanos de las Naciones Unidas organizó una reunión virtual con la alta comisionada para los Derechos Humanos para discutir las implicaciones de covid-19 en los derechos humanos, sin precedentes, en respuesta a la pandemia. Durante la sesión,

15. «Covid-19 and human rights: We are all in this together», Naciones Unidas, abril de 2020, disponible en https://bit.ly/2G6yeki.

16. «Addressing human rights as key to the Covid-19 response», Organización Mundial de la Salud, 21 de abril de 2020, disponible en https://bit.ly/330BPcA.

17. Comité de Derechos Económicos, Sociales y Culturales, Observación General 25, relativa a la ciencia y los derechos económicos, sociales y culturales (artículo 15, párrafos 1 b), 2, 3 y 4, del Pacto Internacional de Derechos Económicos, Sociales y Culturales; $\mathrm{y}$ «Declaración sobre la pandemia de enfermedad por coronavirus (covid-19) y los derechos económicos, sociales y culturales», E/C.12/2020/1, 2020.

18. Por parte de los procedimientos especiales, el primer comunicado de prensa relacionado con covid-19 se emitió el 16 de marzo de 2020, y desde entonces hasta el 6 de agosto de 2020, los procedimientos especiales han emitido 91 declaraciones y comunicados de prensa en total.

19. «El covid-19 plantea un "reto colosal para el liderazgo" que exige una actuación coordinada, declara la alta comisionada ante el Consejo de Derechos Humanos», Naciones Unidas, 9 de abril de 2020, disponible en https://bit.ly/3e2QGXI.

20. «Coronavirus: 'No-one is safe until everyone is safe,' says WHO director», Euronews, 18 de agosto de 2020, disponible en https://bit.ly/3kAvomM. 
en diálogo entre participantes de todo el mundo con la alta comisionada para los Derechos Humanos y grupos de la sociedad civil, se evaluó de manera común la urgencia de proteger los derechos humanos de manera integral. La obligación de los Estados de evitar o mitigar los efectos negativos en el disfrute de los DESCA ha sido destacada en función de todas las obligaciones en materia de derechos humanos ${ }^{21}$ y el deber de dedicar el «máximo de recursos disponibles» ${ }^{22}$ en la lucha contra el covid-19, sin imponer una carga económica adicional a los grupos marginalizados, constituye una constante en los diversos pronunciamientos.

En paralelo, la alta comisionada para los Derechos Humanos, ${ }^{23} \mathrm{e}$ igualmente el Comité de Derechos Humanos de las Naciones Unidas, ${ }^{24}$ han ratificado y recordado los principales parámetros internacionales de control aplicables en los estados de excepción. De esa manera, las entidades internacionales han alertado que las medidas adoptadas por los Estados como respuesta a la pandemia deben ser limitadas en el tiempo, legales, ajustadas a los objetivos definidos según criterios científicos, razonables, estrictamente necesarias y proporcionales, y acordes con los demás requisitos desarrollados en el derecho de los derechos humanos. En el ejemplo simbólico del vínculo entre salud y acceso a la información, cabe destacarse el comunicado del relator especial de las Naciones Unidas sobre la Promoción y Protección del Derecho a la Libertad de Opinión y Expresión. ${ }^{25}$

\section{Estándares regionales: África}

Frente a la pandemia, la Comisión Africana de Derechos Humanos y de los Pueblos ha sido el primer órgano regional en hacer una llamada urgente a los Estados miembros de responder la emergencia sanitaria sobre la base de los derechos humanos y a la luz de su interdependencia. Se publicó un comunicado de prensa el 28 de febrero de 2020 sobre la crisis del virus ${ }^{26}$ y la Declaración del 24 de marzo de 2020 enfatizó que «la

21. Alice Donald y Philip Leach, «Human rights: The essential frame of reference in the global response to Covid-19», Verfassungsblog, 12 de mayo de 2020, disponible en https://bit.ly/333mgAY.

22. «Episode 4: Maximum available resources», Universidad de Nottingham, Making Economic and Social Rights Real Project, capítulo 4, disponible en https://bit.ly/3kPwLxO.

23. «Emergency measures and Covid-19: Guidance», Oficina del Alto Comisionado para los Derechos Humanos, 27 de abril de 2020, disponible en https://bit.ly/343FqpH.

24. «Declaración sobre la suspensión de obligaciones dimanantes del Pacto en relación con la pandemia de covid-19", Comité de Derechos Humanos de las Naciones Unidas, CCPR/C/128/2.

25. «Las pandemias y la libertad de opinión y expresión: Informe del relator especial sobre la promoción y protección del derecho a la libertad de opinión y de expresión», Oficina del Alto Comisionado de las Naciones Unidas para los Derechos Humanos, A/HRC/44/49, 23 de abril 2020, disponible en https://bit.ly/3cvWyYQ.

26. «Press statement of the African Commission on Human \& Peoples' Rights on the Coronavirus (Covid-19)», Comisión Africana de Derechos Humanos y de los Pueblos, 28 de febrero de 2020, disponible en https://bit.ly/3ohqOlj. 
Comisión desea recordar las disposiciones pertinentes de la Carta Africana de Derechos Humanos y de los Pueblos, en particular el artículo 4 sobre el derecho a la vida, el artículo 9 sobre el derecho al acceso a la información y el artículo 16 sobre el derecho a la salud». ${ }^{27}$ En consonancia con ese llamamiento de la Comisión Africana, el Grupo de Trabajo sobre DESC recordó los efectos negativos en la salud, el trabajo, la educación, la alimentación, el agua y la seguridad social, a pesar de la aplicación de las medidas de bienestar social establecidas por varios Estados miembros como parte de su respuesta nacional al covid-19. ${ }^{28}$ El grupo urge a los Estados a adoptar medidas que respeten los tratados regionales e instrumentos internacionales de derechos humanos para garantizar el pleno disfrute de todos los derechos — «durante y después de la crisis»- ${ }^{29} \mathrm{y}$ destaca en particular la situación de los grupos vulnerables y marginados que apenas en circunstancias normales disfrutan del derecho a la vivienda y no tienen un acceso equitativo a la salud, la educación, al agua, al saneamiento, a la alimentación y al trabajo. De manera complementaria, también se constata la dependencia entre los derechos políticos y el derecho a la salud, en específico con referencia al derecho de las elecciones como pilar de la democracia, que debe ser salvaguardado durante la emergencia sanitaria..$^{30}$ En el contexto africano se suma la dimensión de la paz en el entendimiento de la interdependencia e indivisibilidad de derechos humanos, pues en las palabras de Solomon Ayele Dersso, presidente de la Comisión Africana, las previsibles consecuencias socioeconómicas y humanitarias de la crisis pueden incidir en procesos de paz, en tanto son factores generadores de las situaciones de conflictos e inseguridades..$^{31}$

27. "Press statement on human rights based effective response to the novel Covid-19 virus in Africa», Comisión Africana de Derechos Humanos y de los Pueblos, 24 de marzo de 2020, disponible en https:// bit.ly/3mW49Vn.

28. Estas medidas incluyen la distribución gratuita de alimentos, el suministro de agua gratuito y mejorado, la asistencia financiera, la reducción temporal de las facturas de electricidad y el suministro de alojamiento para las personas sin hogar. «Press release on the impact of the Covid-19 pandemic on economic, social and cultural rights in Africa», Comisión Africana de Derechos Humanos y de los Pueblos, 4 de junio de 2020, disponible en https://bit.ly/3kVlEU8.

29. «Press release».

30. «La Comisión afirma que el derecho a una elección regular, libre, justa y creíble es la norma democrática más sagrada que sirve de medio principal para el ejercicio del derecho soberano de un pueblo al autogobierno en virtud del artículo 20 de la Carta Africana y el derecho de las personas a participar en los asuntos públicos en virtud del artículo 13 de la Carta Africana. La celebración de elecciones dentro de los parámetros establecidos constitucionalmente y las normas de derechos humanos aplicables, incluidas las previstas en la Carta Africana sobre Democracia, Elecciones y Gobernanza, es un requisito previo para el mantenimiento de un sistema de gobierno democrático, responsable y receptivo». «Statement of the African Commission on Human and Peoples' Rights on elections in Africa during the Covid-19 pandemic», Comisión Africana de Derechos Humanos y de los Pueblos, 22 de julio de 2020, disponible en https://bit.ly/2G5hNoA.

31. «Statement of Commissioner Solomon Ayele Dersso, Chairperson of the African Commission on 


\section{Estándares regionales: Europa}

En Europa se deja de manifiesto la tendencia hacia el compromiso con los principios de interdependencia e indivisibilidad en esta renovada lectura mediante múltiples iniciativas de los órganos regionales y sus representantes, guías prácticas y recomendaciones para los Estados, así como pronunciamientos a favor de la protección reforzada de los grupos vulnerables. Se ha recordado, en palabras del alto representante Josep Borrell, «que todos los derechos humanos son universales, indivisibles e interdependientes y que están interrelacionados. [...] Nos comprometemos a velar por que nuestra respuesta salvaguarde la dignidad y los derechos humanos de todas las personas sin discriminación de ningún tipo».32 Eamon Gilmore, representante especial de la Unión Europea para los Derechos Humanos, afirmó categóricamente que los derechos humanos están en el centro de la batalla contra el covid-19. En términos elocuentes reivindica esta nueva mirada de la interdependencia e indivisibilidad al afirmar que

proteger y preservar la vida es el propósito principal de esta lucha. Sin el derecho a la vida, es imposible ejercer otros derechos. Para proteger la vida, debemos reivindicar el derecho a la salud. Este, a su vez, depende no solo del acceso a la atención médica, sino también del derecho al agua potable y al saneamiento, a una nutrición adecuada y a un ambiente seguro y saludable. También requiere acceso a la información, para que las personas estén facultadas para proteger su propia salud y la de los demás. $Y$ en esta crisis sanitaria, que requiere una respuesta colectiva y la cooperación de las personas en todas partes, el respeto por la sociedad civil es más importante que nunca. Todos los derechos humanos son interdependientes e indivisibles. ${ }^{33}$

Por su parte, el secretario general del Consejo de Europa lanzó como guía de buena práctica un conjunto de herramientas, ${ }^{34}$ para recordar a los Gobiernos de toda Europa garantizar el respeto de los derechos humanos y derechos fundamentales -llamando a la protección del derecho a la educación, de la libertad de expresión,

Human and Peoples' Rights at the Africa Dialogue Series organized by the United Nations Office of the Special Advisor on Africa and the African Union under the theme Covid-19 and silencing the guns in Africa: Challenges and opportunities, 20-22 May 2020», Comisión Africana de Derechos Humanos y de los Pueblos, 26 de mayo de 2020, disponible en https://bit.ly/34362qE.

32. «Declaración del alto representante, Josep Borrell, en nombre de la Unión Europea, sobre los derechos humanos en tiempos de pandemia de coronavirus», Consejo de la Unión Europea, 5 de mayo de 2020, disponible en https://bit.ly/36hdL7c.

33. Eamon Gilmore, «La lucha contra covid-19 es una batalla por los derechos humanos», Delegación de la Unión Europea en Cuba, 17 de abril de 2020, disponible en https://bit.ly/2FXqdhT.

34. "Respecting democracy, rule of law and human rights in the framework of the Covid-19 sanitary crisis: A toolkit for member states», Consejo de Europa, SG/Inf(2020)11, 7 de abril de 2020, disponible en https://bit.ly/2EKNJOw. 
de la intimidad y los datos, de los grupos vulnerables y contra la discriminación-y garantizar la democracia y el Estado de derecho durante la crisis de covid-19. No se deja de lado la determinación de los límites del alcance y la duración de las medidas de emergencia. ${ }^{35}$

En lo concerniente a los impactos negativos de la pandemia sobre determinados grupos, vale poner de relieve la nota del representante especial del secretario general sobre Migración y Refugiados del Consejo de Europa, junto con la Agencia de Derechos Fundamentales de la Unión Europea, que insiste en las principales salvaguardias de los derechos fundamentales de los refugiados, solicitantes de asilo y migrantes aplicables en las fronteras exteriores de sus Estados miembros. ${ }^{36}$ El Comité Europeo de Derechos Sociales ofrece asimismo orientación a través de su sitio web dedicado a los «Derechos sociales en tiempos de pandemia: Covid-19 y la Carta Social Europea», y examina las medidas adoptadas por los Estados en lo que respecta a sus obligaciones en materia de derechos sociales.

\section{Estándares regionales: América Latina}

Frente a la pandemia, la Corte IDH y la CIDH han reiterado el principio de interdependencia e indivisibilidad de los derechos humanos y han denotado la protección reforzada para atender a las personas y grupos en situación de vulnerabilidad, reflejando de manera reiterativa los casos de interseccionalidad (Góngora-Mera, 2020). Sin duda, el Sistema Interamericano también ha subrayado que las medidas que puedan afectar o restringir el goce y ejercicio de derechos humanos deben cumplir con los principios de temporalidad, legalidad, razonabilidad, necesariedad, proporcionalidad y ser conformes a criterios científicos.

El ejercicio del mandato transformador se ha concretizado asimismo a través de los comunicados conjuntos con la Relatoría Especial sobre Derechos Económicos, Sociales, Culturales y Ambientales (REDESCA) y la Relatoría Especial para la Libertad de Expresión (RELE), que han dejado sentado, una vez más, el orden público interamericano. Como una alerta temprana y enmarcado en el sistema multinivel, el primer comunicado de prensa señala que los Gobiernos deben promover y proteger el acceso y la libre circulación de la información durante la pandemia ${ }^{37}$ seguido del llamado a asegurar las perspectivas de protección integral de los derechos humanos

35. «Human rights and Covid-19», European Trade Union Confederation, 7 de mayo de 2020, disponible en https://bit.ly/2HDQqT3.

36. «Fundamental rights of refugees and migrants at European borders», European Union Agency for Fundamental Rights, 27 de marzo de 2020, disponible en https://bit.ly/349w4IX.

37. «Covid-19: Los Gobiernos deben promover y proteger el acceso y la libre circulación de la información durante la pandemia: Expertos internacionales», Organización de los Estados Americanos, R58/20, 19 de marzo de 2020, disponible en https://bit.ly/2G7px9B. 
y de la salud pública frente a la pandemia ${ }^{38} \mathrm{y}$, de modo consecutivo con más de una docena de comunicados, ha advertido la urgencia de proteger los derechos de los grupos más vulnerables. Con un mecanismo novedoso, el 27 de marzo de 2020, la Comisión anunció la instalación de su Sala de Coordinación y Respuesta Oportuna e Integrada a la crisis en relación con la pandemia del covid-19 (SACROI covid-19), para monitorear el impacto en los derechos humanos y, en particular, en los grupos en situación de vulnerabilidad en los 35 países de las Américas.

El 10 de abril se aprobó la Resolución 01/2020, sobre Pandemia y Derechos Humanos, con 85 recomendaciones destinadas a los Estados, a la luz de los estándares interamericanos y del principio pro persona. La resolución enfatiza el deber de los Estados de adoptar un enfoque centrado en los derechos humanos en toda estrategia, política o medida estatal de enfrentamiento a la pandemia del covid-19 y sus consecuencias. Ya un primer balance, impacto y resultados alcanzados se presentan en el comunicado de prensa del 16 de mayo de 2020. La CIDH, el 27 de julio de 2020, adoptó además la Resolución 04/2020, sobre Derechos Humanos de las Personas con Covid-19, con el propósito de visibilizar, mediante 52 directrices, que los pueblos de las Américas continúan estando extremadamente afectadas por la pandemia global, y que quienes han contraído el virus corren riesgos de no ver salvaguardados sus derechos humanos.

La Declaración 1/20 emitida por la Corte Interamericana, titulada «Covid-19 y derechos humanos: Los problemas y desafíos deben ser abordados con perspectiva de derechos humanos y respetando las obligaciones internacionales», insta a los Estados a adoptar e implementar medidas que estén dentro del marco del Estado de derecho, con pleno respeto de los instrumentos interamericanos para la protección de los derechos humanos y los estándares desarrollados por la jurisprudencia de la Corte. En cuanto a los derechos económicos, sociales, culturales, y ambientales, se publicó una infografía sobre «Covid-19 y el derecho a la salud», que sintetiza las obligaciones estatales a la hora de limitar los derechos económicos, sociales y culturales, y la protección reforzada de los grupos vulnerables. Asimismo, se publicó el nuevo Cuadernillo de Jurisprudencia 28 sobre el derecho a la salud, el cual versa sobre el debate universal de la pandemia de covid-19.

Pionera, una vez más, la Corte IDH, a través de la resolución de la presidenta, Elizabeth Odio Benito, dictó medidas urgentes en el marco del caso Vélez Loor con Panamá. Estas medidas se originan a raíz de la solicitud de las víctimas para que la Corte le requiera a Panamá la implementación de medidas de protección respecto de personas migrantes detenidas en el centro La Peñita, para proteger sus derechos a la

38. «La CIDH y su REDESCA instan a asegurar las perspectivas de protección integral de los derechos humanos y de la salud pública frente a la pandemia del covid-19», Organización de los Estados Americanos, 6o/2020, 20 de marzo de 2020, disponible en https://bit.ly/3n1UoXp. 
vida, salud e integridad en el marco de la pandemia. Al analizar la situación, se tuvo en cuenta la citada Declaración 1/20, así como el corpus iuris interamericano, por lo que le requirió al Estado, entre otros, asegurar, «de forma inmediata y efectiva, el acceso a servicios de salud esenciales sin discriminación a todas las personas que se encuentran en las estaciones de recepción migratoria La Peñita y Laja Blanca, incluyendo detección temprana y tratamiento del covid-19».

En la idea de profundizar la indivisibilidad e interdependencia, la Corte IDH da cuenta de su jurisprudencia en materia de estados de excepción y suspensión de derechos con la reedición del Cuadernillo de Jurisprudencia 26, «Restricción y suspensión de derechos humanos», que acentúa que ningún derecho puede ser suspendido sin cumplir con las condiciones estrictas del artículo 27.1 de la CADH. Otras iniciativas valiosas son el Centro de Información Covid-19 y Derechos Humanos, un espacio de difusión en la web, y el «Diálogo entre las tres Cortes Regionales de Derechos Humanos», que permitió evidenciar "que en los tres continentes esta pandemia está afectando desproporcionalmente a ciertas personas históricamente discriminadas, como mujeres, migrantes, adultas mayores, entre otras»..$^{39}$

Surge entonces la cuestión de verificar si, a nivel nacional, tomando Argentina como caso de estudio, se puede perfilar una respuesta que se aproxima hacia esta reinterpretación de la indivisibilidad e interdependencia a la inversa y reforzada para los más vulnerables. Se pondrán de relieve tres rasgos: a saber, la visión compartida en cuanto a la reinterpretación, la significación de la protección reforzada de los más marginalizados enclavada en los estándares universales y regionales y ciertas buenas prácticas como reflejo de la internacionalización e interamericanización de los órdenes nacionales.

Partiendo del Plan Operativo de preparación y respuesta al covid- $19,{ }^{40}$ se constata que el Estado nacional ha tomado las medidas por parte de todas las áreas del Gobierno y ha destacado que corresponden a la lógica de la complementariedad de las distintas políticas y con un abordaje desde los efectos de la pandemia en los derechos fundamentales de la población. Esta respuesta confirma el argumento sostenido en tanto no solo se requiere reconocer la interdependencia y la indivisibilidad a la inversa, sino que también van más allá en el sentido de comprometerse en «respuestas integrales» entre las diferentes entidades de la autoridad pública. El Comité de Ética y Derechos Humanos en Pandemia Covid-19 fue creado ad hoc y ad honorem según la Resolución 1.117/2020 (firmada por el ministro de Salud de la Nación), la

39. "Covid-19 y derechos humanos: Diálogo entre las tres cortes regionales de derechos humanos», Corte Interamericana de Derechos Humanos, 54/2020, 13 de julio de 2020, disponible en https://bit. ly/2GfeWsT.

40. «Plan operativo de preparación y respuesta al covid-19», Gobierno de Argentina, Ministerio de Salud, disponible en https://bit.ly/3iilLHG. 
cual declara necesario tal comité ante las múltiples incertidumbres que plantea el covid-19. El nuevo órgano tiene como función fomentar el diálogo interdisciplinario entre la política sanitaria, la ciencia y la ética para generar recomendaciones en apoyo de las tareas destinadas al cuidado de la salud pública, a partir de la protección de los derechos fundamentales de la población y considerando las recomendaciones de la Organización Mundial de la Salud. Invocan la filosofía del Estado constitucional, convencional, democrático, social y de derecho que rige la nación argentina y el derecho internacional de los derechos humanos.

En concordancia con el ejemplo simbólico del vínculo entre salud y acceso a la información, Argentina decretó que el Ministerio de Salud informaría diariamente sobre las «zonas afectadas» y la situación epidemiológica. Respecto de la propagación, contención y mitigación del virus, destinó un sitio web para dar a conocer las medidas obligatorias y recomendaciones y la presentación de un reporte matutino y uno vespertino transmitidos por los medios de comunicación. ${ }^{41}$ Para la protección reforzada de un grupo vulnerable, se configuró una línea gratuita exclusiva para que las personas mayores puedan hacer consultas sobre el nuevo coronavirus y acceder a la información sobre las recomendaciones específicas para el cuidado de su salud. ${ }^{42}$ En la configuración del ius commune se inscriben buenas prácticas como la defensa del derecho a la vivienda de las personas residentes en barrios populares, destacada por expertos de Naciones Unidas, ${ }^{43}$ o la señalada por la $\mathrm{CIDH}^{44}$ cuando se exceptuó de la suspensión de los plazos administrativos —establecida por los DNU 298/20 y sus prórrogas-, a los trámites previstos por la Ley 27.275 (de Acceso a la Información

41. Véase el Decreto 260/2020, del 12 de marzo de 2020; y la Resolución 568/2020 del Ministerio de Salud, del 14 de marzo de 2020.

42. El Programa de Atención Médica Integral (PAMI) del Gobierno argentino habilitó una línea gratuita exclusiva (138, opción 9) y una campaña en redes sociales con herramientas audiovisuales accesibles.

43. En el caso de Argentina, se pueden destacar como buenas prácticas «los Decretos Nacionales $319 / 2020$ y $320 / 2020$, que suspenden los desalojos por falta de pago, prorrogan la vigencia de contratos de alquiler y congelan las cuotas de créditos hipotecarios, ya que pueden contribuir a reducir la vulnerabilidad frente a la pandemia de covid-19». Véase la comunicación conjunta enviada por el Relator Especial sobre los derechos humanos al agua potable y el saneamiento; el Relator Especial sobre el derecho de toda persona al disfrute del más alto nivel posible de salud física y mental; el Relator Especial sobre una vivienda adecuada como elemento integrante del derecho a un nivel de vida adecuado; y el Relator Especial sobre la extrema pobreza y los derechos humanos, Naciones Unidas, 24 de junio de 2020, disponible en https://bit.ly/30yng8T; Decreto 319/2020, Boletín Oficial de la República de Argentina, 29 de marzo de 2020, disponible en https://bit.ly/2Twze4v; Decreto 320/2020, Boletín Oficial de la República de Argentina, 29 de marzo de 2020, disponible en https://bit.ly/3kBzMSL.

44. «CIDH presenta balance, impactos y resultados alcanzados por su Sala de Coordinación y Respuesta Oportuna e Integrada a Crisis para la Pandemia de Covid-19", Organización de los Estados Americanos, 114/20, 16 de mayo de 2020, disponible en https://bit.ly/3jbIQNl. 
Pública) y la Ley 25.326 (de Protección de Datos Personales). ${ }^{45}$ En la interamericanización del derecho al agua, ${ }^{46}$ se recoge la decisión de un juez de distrito que ordenó al Gobierno de la Ciudad de Buenos Aires preparar un plan de contingencia que establezca un cronograma detallado para el suministro de agua potable en comunidades sin acceso al agua del grifo y asegurar el suministro de agua en tanques de agua. En este ámbito, la sentencia de la Corte IDH en el caso Lhaka Hohnat con Argentina define los contornos del derecho humano al agua, enriquecido con los estándares universales y nacionales.

\section{Renovación del discurso sobre indivisibilidad e interdependencia: El potencial transformador pos covid-19}

El impacto dramático del covid-19 y su naturaleza multidimensional impulsan una época de profunda transformación y reinvención, marcada por decisiones cruciales. Habrá un mundo pre covid-19 y un mundo pos covid-19.

Existe desde hace más de siete décadas una visión compartida en el mundo sobre el sistema integral de derechos humanos basada en al entendimiento de que, para garantizar la dignidad de la persona humana, debían alcanzarse todos los derechos humanos (Minkler y Sweeney, 2011). Los límites de conceptualizarlos a la luz de su interdependencia e indivisibilidad en la lectura tradicional ya se esbozaron en el mundo pre covid-19. Entre distintos discursos, se alude a la incorporación de una perspectiva de justicia social, sobre todo para enfrentar el reto de atender las necesidades de grupos y poblaciones vulnerables (Nueves-Silva, Martins y Heller, 2019); también desde el punto de vista de la práctica, con referencia especial al derecho a la salud y la falta de entenderlo en su integridad con los demás derechos, ${ }^{47} \mathrm{o}$ sobre la necesidad de crear herramientas de monitoreo adecuados para poder entender mejor las consecuencias de violaciones de derechos interdependientes (Bouchard y Meyer-Bisch, 2016), lo que ha empujado hacia un cambio dogmático y práctico.

La pandemia nos ha dado la evidencia de la necesidad de repensar los alcances de los principios de indivisibilidad e interdependencia y, más allá, nos insta a repensarlos a la inversa y reforzar la protección de las personas y grupos en situación de vulnerabilidad, con mayor énfasis en la igualdad aproximándonos desde los déficits estructurales. El contexto actual puede ofrecer un punto de partida hacia un sistema

45. Resolución 70/2020, Agencia de Acceso a la Información Pública.

46. Mariela Morales Antoniazzi, «How a landmark decision from the Inter American Court on Argentina can help shape a response to Covid-19 and the right to water: New momentum for the ius commune», EJIL Talk, 29 de mayo de 2020, disponible en https://bit.ly/3cF5 Kdr.

47. Dainius Pūras, «Challenges in promoting the interdependence of all human rights», Health and Human Rights Journal, 18 de diciembre de 2019, disponible en https://bit.ly/3kQ26Ac. 
más integrativo, resistente, resiliente y sostenible, con la debida priorización de los grupos más vulnerables.

Desde la perspectiva de los derechos humanos, una «disrupción» como el covid-19 podría generar una profunda transformación, ya que la pandemia constituye el mayor reto enfrentado por la humanidad desde hace décadas. Categorizar la pandemia como «disrupción» permite apuntar hacia un cambio de gran alcance en las prácticas de derechos humanos en cuanto a la intensidad y urgencia de la conmoción en el sistema de derechos humanos. ${ }^{48}$ Como surgió la creación de las Naciones Unidas y el derecho internacional de los derechos humanos como respuesta a las atrocidades de la Segunda Guerra Mundial, las respuestas ante el covid-19 pueden significar un momento refundacional, en coherencia con la llamada «Contribución del respeto de todos los derechos humanos y las libertades fundamentales al logro de los propósitos y la defensa de los principios de la Carta de las Naciones Unidas», ${ }^{49}$ emitida en julio de 2020 por la Asamblea General. Subrayar la indivisibilidad de la salud y los otros derechos no es solo una cuestión teórica, sino que tiene un sustento práctico esencial que interpela la eficacia de la autoridad pública $^{50}$ y que impone límites jurídicos a la actuación estatal en su ejercicio de autoridad (Hostmaelingen y Bentzen, 2020).

En este orden de ideas, es oportuno pensar en una refundación de los derechos humanos, buscar enfoques con un potencial transformador, entre ellos, los conceptos de ius commune y comunidad de práctica en derechos humanos. Es necesario que el discurso académico se base en el reconocimiento de todos los derechos humanos para articular las respuestas a nivel global, regional y nacional frente a la pandemia.

A la luz de los desafíos estructurales que deben enfrentarse, agravados y con sus impactos exacerbados en los derechos humanos, se puede reiterar que la efectividad de los DESCA está absolutamente condicionada a la efectividad de los derechos ci-

\footnotetext{
48. Según Oona Hathaway (2002: 2.003-2.004), se puede detallar: «Las grandes crisis del sistema proporcionan ventanas de oportunidad limitadas para efectuar grandes cambios en el sistema. De hecho, cuando se producen cambios importantes en las prácticas de derechos humanos, a menudo se debe a ese acontecimiento». La idea un momento fundador de cambio institucional tiene sus raíces en el institucionalismo histórico. Este último se centra en las instituciones formales e informales y considera su evolución tras una trayectoria estable en tiempos normales. Una cepa del análisis de esta teoría sostiene que las coyunturas circulares son momentos particulares de la historia en los que se levantan o se suavizan las limitaciones habituales de la acción, lo que incluye también el peso relativo del organismo frente a la estructura durante esos episodios». Véase, sobre cambios institucionales, Mahoney y Kathleen (2010); y para un estudio sobre Perú y el reforzamiento de derechos humanos por una ventana de oportunidad, Root (2009).

49. A/HRC/44/L.17/Rev.1 con ocasión del $75 .^{\circ}$ aniversario de las Naciones Unidas, 14 de julio de 2020, disponible en https://bit.ly/35JwoP9.

50. Lisa Forman, «The evolution of the right to health in the shadow of Covid-19", Health and Human Rights Journal, 2 de abril de 2020, disponible en https://bit.ly/3cFcDfi.
} 
viles y políticos, y la efectividad de los derechos civiles y políticos en su plenitud está absolutamente condicionada a la efectividad de los DESCA.

El mundo pos covid-19 impone abordar los hitos y mitos en lo atinente a la pobreza como una condición generalizada de violación de múltiples derechos humanos; en paralelo, convoca a apropiarse de todo el acervo normativo, jurisprudencial y discursivo que se ha venido gestando progresivamente:

Tres ejes son clave, a saber, la noción de pobreza desde su alcance multidimensional y rechazar la conceptualización en términos de mera escasez de ingresos y más bien asumir el enfoque centrado en la capacidad como el puente conceptual entre la pobreza y los derechos humanos; un segundo hito es «re-advertir» el grado en que la pobreza afecta también la capacidad de las personas para disfrutar de sus derechos civiles y políticos porque les niega la voz política, crea obstáculos para reclamar su situación y se le privación de sus capacidades para disfrutar de sus derechos humanos. Y un tercer hito deviene de respaldar una orientación más sustantiva de la igualdad que permita a las Cortes, como actores del proceso democrático, considerar más adecuadamente los vínculos entre pobreza y discriminación y ampliar el espectro de los conceptos de vulnerabilidad y discriminación interseccional (Morales Antoniazzi, 2021).

Para América Latina, resulta esencial repotenciar el discurso alusivo a la protección prioritaria de las personas que viven en la pobreza, en el entendido de que los órganos del sistema interamericano ejercen un mandato transformador a la luz de la CADH y su interpretación como instrumento vivo.

Son conocidas las voces críticas en cuanto a las limitaciones del discurso de los derechos humanos (Moyn, 2018). No obstante, existen estudios que preponderan el rol que pueden desempeñar en el combate de la desigualdad las sentencias de tribunales nacionales, cuando actúan en la interpretación de las constituciones nacionales y deciden a favor de la promoción de la igualdad a partir del discurso de los derechos humanos (Versteeg, 2020). En el ámbito del sistema interamericano, la academia pone de relieve la reconceptualización de la igualdad y describe la tendencia a aproximarse a una comprensión de la igualdad como «antisubordinación», con miras a afrontar los problemas estructurales profundamente arraigados (Aldao, Clérico y Ronconi, 2017).

Las desigualdades y los impactos discriminatorios de la pandemia para las personas y grupos en vulnerabilidad traen consigo un llamado a la academia y a la práctica para buscar una transformación hacia sistemas más justos, con inclusión social y política..$^{51}$ Ya se encuentran estudios destinados a evaluar los escenarios y las soluciones

51. Véanse, por ejemplo, Olivier de Schutter, «La crisis por covid-19 destaca la urgente necesidad de transformar la economía global, dice nuevo experto de la ONU en pobreza», Oficina del Alto Comi- 
potenciales, los puntos de vista multidisciplinarios y los efectos de las medidas frente al covid-19 (Buheji y otros, 2020), así como el perfilamiento de perspectivas acerca de un estado de bienestar con base en un nuevo pacto social (fiscal, social y productivo), con la universalización de acceso a los derechos, ingreso básico universal y un sistema de cuidados. ${ }^{52}$ Son solo ejemplos que dan cuenta del renovado interés académico en torno a dar visibilidad a los invisibilizados.

\section{Comentarios finales}

Los retos actuales revelan el potencial de los derechos humanos como lenguaje para guiar a los Estados hacia el cumplimiento de los estándares que conforman la base del nuevo ius commune pospandemia. No solo es claro un entendimiento holístico de los derechos humanos, sino también su aplicación práctica en la inaplazable lucha contra la pobreza y, en particular, en la atención a las personas y grupos en situación de vulnerabilidad.

A la luz de los desafíos estructurales que deben enfrentarse, agravados y con sus impactos exacerbados en los derechos humanos, se puede reiterar que la efectividad de los DESCA está absolutamente condicionada a la efectividad de los derechos civiles y políticos, mientras que la efectividad de los derechos civiles y políticos en su plenitud está absolutamente condicionada a la efectividad de los DESCA. Algunos ejes transversales para enfrentar los desafíos estructurales que pueden identificarse de manera enunciativa en torno a la desigualdad socioeconómica, la discriminación histórica y la debilidad institucional, serían, en lo socioeconómico:

- El deber de los Estados de adoptar un enfoque centrado en los derechos humanos en toda estrategia, política o medida estatal de enfrentamiento a la pandemia de covid-19 y sus consecuencias.

- Potencializar los DESCA, en tanto los Estados deben asignar especial prioridad a la protección de los derechos a la vida y la salud, mediante la adopción de medidas inmediatas y urgentes, con la debida diligencia, respaldando el

sionado de Naciones Unidas para los Derechos Humanos, 1 de mayo de 2020, disponible en https://bit. ly/33aNq99; «Informe sobre el impacto económico en América Latina y el Caribe de la enfermedad por coronavirus (covid-19)», Cepal, marzo de 2020, disponible en https://bit.ly/3jd7Vay.

52. Alicia Bárcena, secretaria ejecutiva de la Comisión Económica para América Latina y el Caribe (Cepal), en una reunión titulada «Hacia una nueva economía global: Encuentro universitario ministerial». Varios países muestran indicadores que han dado ciertos pasos en esta dirección. Algunos de los países en América Latina aumentaron la protección social desde el inicio de la crisis del coronavirus y adoptaron bonos para las familia en vulnerabilidad (Argentina, Colombia, Perú, entre otros). Véase «El número de niños en hogares pobres puede aumentar en 86 millones debido al coronavirus», Noticias ONU, 28 de mayo de 2020, disponible en https://bit.ly/33ayhVf. 
deber del Estado de prevenir y mitigar los daños, y poniendo a disposición el máximo de los recursos disponibles para la efectividad del derecho a la salud, bajo una concepción integral.

- Las acciones, programas y políticas públicas de los Estados deben atender la mejor evidencia científica.

- Los Estados deben exigir y fiscalizar que las empresas respeten los derechos humanos.

Ante la discriminación histórica:

- Para los grupos en especial situación de vulnerabilidad rige el deber reforzado de protección estatal a las personas mayores, las personas privadas de libertad, las mujeres, los pueblos indígenas, las personas migrantes y refugiadas, los niños, niñas y adolescentes, las personas LGBTI, los afrodescendientes y las personas con discapacidades.

- Adopción de programas de prevención a la pandemia específicos (por ejemplo, en los idiomas tradicionales de los pueblos indígenas, canales alternativos de denuncia para las mujeres, protocolos específicos para las personas LGBTI y medidas alternativas de privación de libertad).

A la luz de los dilemas de la institucionalidad democrática:

- Los Estados, en cualquier restricción de derechos y libertades, deben observar estrictamente los principios de legalidad, proporcionalidad, necesaridad y temporalidad, a fin de cumplir con las finalidades legítimas de proteger a la salud.

- La importancia del funcionamiento de los poderes, sobre todo los procedimientos judiciales idóneos para la protección de derechos y libertades, así como la garantía de los derechos a la libertad de expresión y el acceso a la información, los cuales son pilares esenciales del régimen democrático.

- La vigilancia digital debe limitarse estrictamente a la rigurosa protección de los derechos individuales, al principio de no discriminación y a las libertades fundamentales.

Así como la pandemia advierte que compartimos las mismas amenazas, miedos y riesgos, también advierte que compartimos la misma humanidad y la capacidad de lograr que todos y todas sean libres e iguales en dignidad y derechos hacia el proceso de reconstrucción de sociedades más resilientes, inclusivas y justas. 


\section{Reconocimiento}

La versión original de este artículo fue publicada en Covid-19 y derechos humanos: La pandemia de la desigualdad, editado por Juan Pablo Bohoslavsky (Buenos Aires: Biblos, 2020).

\section{Referencias}

AldaO, Martín, Laura Clérico y Liliana Ronconi (2017). «A multidimensional approach to equality in the Inter-American context: Redistribution, recognition, and participatory parity». En Armin von Bogdandy, Eduardo Ferrer Mac-Gregor, Mariela Morales Antoniazzi, Flávia Piovesan y Ximena Soley (editores), Transformative constitutionalism in Latin America: The emergence of a new ius commune. Oxford: Oxford University Press.

Bouchard, Johanne y Patrice Meyer-Bisch (2016). «Intersectionality and interdependence of human rights: Same or different?». The Equal Rights Review, 16: 186203. Disponible en https://bit.ly/36e $51 \mathrm{Pl}$.

Buheji, Mohamed, Katiane da Costa Cunha, Godfred Beka, Bartola Mavrić, Yuri Leandro do Carmo de Souza, Simone Souza da Costa Silva, Mohammed Hanafi y Tulika Chetia Yein (2020). "The extent of Covid-19 pandemic socio-economic impact on global poverty: A global integrative multidisciplinary review». American Journal of Economics, 10 (4): 213-224. DOI: 10.5923/j.economics.20201004.02.

Carmona Cuenca, Encarna (2017). «Derechos sociales de prestación y obligaciones positivas del Estado en la jurisprudencia del Tribunal Europeo de Derechos Humanos». Revista de Derecho Político, 100: 1.209-1.238. DOI: 10.5944/ rdp.100.2017.20731.

FARER, Tom (1997). «The rise of the Inter-American human rights regime: No longer a unicorn, not yet an ox». Human Rights Quarterly, 19 (3): 510-546. Disponible en https://bit.ly/36g3AQg.

Ginsburg, Tom y Mila Versteeg (2020). «The bound executive: Emergency powers during the pandemic». Virginia Public Law and Legal Theory Research Paper, 52. Disponible en https://bit.ly/3n1uilx.

Góngora-Mera, Manuel Eduardo (2020). «Discriminación en clave interseccional: Tendencias recientes en la jurisprudencia de la Corte Interamericana de Derechos Humanos». En Mariela Morales Antoniazzi, Liliana Ronconi y Laura Clérico (coordinadoras), Interamericanización de los DESCA: El caso Cuscul Pivaral de la Corte IDH (pp. 399-427). Ciudad de México: Instituto de Estudios Constitucionales del Estado de Querétaro.

Gros EsPiell, Héctor (1986). Los derechos económicos, sociales y culturales en el sistema interamericano. San José: Libro Libre. 
Hathaway, Oona (2002). «Do human rights treaties make a difference?». Yale Law Journal, 111: 1.935-2.042. Disponible en https://bit.ly/3oiwZWp.

Hostmaelingen, Njal y Heidi Bentzen (2020). «How to operationalise human rights for Covid-19 measures». BMJ Global Health, 5 (3): eoo3048. DOI: 10.1136/ bmjgh-2020-003048.

KocH, Ida Elisabeth (2009). Human rights as indivisible rights: The protection of socioeconomic demands under the European convention on human rights. Leiden y Boston: Martiners Nijhoff.

Mahoney, James y Kathleen Thelen (2010). Explaining institutional change: Ambiguity, agency, and power. Cambridge: Cambridge University Press.

Minr, Anja y Mark Gibney (2014). The SAGE handbook of human rights. Los Ángeles: SAGE.

MinkLER, Lanse y Shawna Sweeney (2011). "On the indivisibility and interdependence of basic rights in developing countries». Human Rights Quarterly, 33 (2): 351-396. DOI: 10.1353/hrq.2011.0017.

Morales Antoniazzi, Mariela (2021). «La Corte IDH y la interamericanización del test democrático frente al covid-19». En Sergio García Ramírez y César Astudillo (coordinadores), La Corte Interamericana de Derechos Humanos: Organización, funcionamiento y trascendencia. Ciudad de México: IIJ-UNAM, Tirant lo Blanch (en imprenta).

Moyn, Samuel (2018). Not enough: Human rights in an unequal world. Cambridge: Harvard University Press.

Neves-Silva, Priscila, Giselle Isabele Martins y Léo Heller (2019). «Human rights' interdependence and indivisibility: A glance over the human rights to water and sanitation». BMC International Health and Human Rights, 19 (14). DOI: 10.1186/ S12914-019-0197-3.

Piovesan, Flávia, Mariela Morales Antoniazzi y Erika Montero (2019). «Avances en la protección de las personas mayores en el sistema interamericano: Perspectivas y retos para la efectividad de sus derechos». En Mariela Morales Antoniazzi y Laura Clérico (coordinadoras), Interamericanización del derecho a la salud: Perspectivas a la luz del caso Poblete de la Corte IDH. Ciudad de México: UNAM.

Root, Rebecca K. (2009). «Through the window of opportunity: The transitional justice network in Peru». Human Rights Quarterly, 31 (2): 452-473. DOI: 10.1353/ hrq.0.0073.

SsenYonjo, Manisuli (2016). Economic, social and cultural rights in International Law. Portland: Hart.

Versteeg, Mila (2020). «Can rights combat economic inequality?». Harvard Law Review, 133 (6): 2.017-2.06o. Disponible en https://bit.ly/2GobKSp. 
VoN Bogdandy, Armin y René Urueña (2020). «International transformative constitutionalism in Latin America». American Journal of International Law, 114 (3): 403-442. DOI: 10.1017/ajil.2020.27.

Williams, Andrew (2013). «The European Convention on Human Rights, the EU and the UK: Confronting a heresy». European Journal of International Law, 24 (4): 1.157-1.185. DOI: 10.1093/ejil/chto59.

\section{Sobre las autoras}

Flávia Piovesan es profesora de Derecho Constitucional y Derechos Humanos en la Facultad de Derecho de la Pontificia Universidad Católica de São Paulo, Brasil. Comisionada de la Comisión Interamericana de Derechos Humanos. Su correo electrónico es fpiovesan@oas.org.

Mariela Morales Antoniazzi es investigadora senior del Instituto Max Planck de Derecho Público Comparado y Derecho Internacional de Heidelberg. Coordina el proyecto Ius Constitutionale Commune en América Latina (ICCAL). Su correo electrónico es morales@mpil.de. 
El Anuario de Derechos Humanos es una publicación semestral de referencia y consulta en materia de derechos humanos y campos afines. Busca ser un espacio de discusión de los temas centrales en el ámbito nacional e internacional sobre derechos humanos. Es publicado desde 2005 por el Centro de Derechos Humanos de la Facultad de Derecho de la Universidad de Chile.

\author{
EDITORA GENERAL \\ Claudia Iriarte Rivas \\ ciriarter@derecho.uchile.cl \\ EDITORA DE ESTE NÚMERO \\ Liliana Galdámez Zelada \\ lgaldamez@derecho.uchile.cl \\ SITIO WEB \\ anuariocdh.uchile.cl \\ CORREO ELECTRÓNICO \\ anuario-cdh@derecho.uchile.cl \\ LICENCIA DE ESTE ARTÍCULO
}

Creative Commons Atribución Compartir Igual 4.o Internacional

\author{
कै \\ La edición de textos, el diseño editorial \\ y la conversión a formatos electrónicos de este artículo \\ estuvieron a cargo de Tipográfica \\ (www.tipografica.io)
}

